\title{
Introduction to the Special Issue on Quest 2017
}

This special issue is based on selected papers presented and published at the 14th International Conference on Quantitative Evaluation of Systems, QEST 2017, held in Berlin on September 5-7, 2017. QEST is one of the main conferences on the quantitative evaluation and verification of computer systems and networks, based on stochastic models and measurements. QEST covers both methodological approaches, including the development of new algorithms, methods to analyze systems, and measurement-based studies, tools, and applications of tools and theory to the design of systems in a broad sense. Systems of interest include computer hardware and software architectures, communication systems, embedded systems, infrastructural systems, and biological systems.

The guest editors of this special issue served as Program Chairs of QEST 2017 and were responsible for the proceedings of the conference. The conference received 58 submissions and accepted 20 full papers and 4 tool papers. Out of these papers, the top 8 papers were selected in collaboration with the editor-in-chief of TOMACS and invited to submit to the special issue. One of them declined, which resulted in 7 submissions. The guest editors requested the authors to prepare a document outlining changes and extensions to their original QEST paper, also taking into account the reviewer comments received for the conference submission. The special-issue papers were peer reviewed by a minimum of three reviewers. Most underwent two rounds of reviews, with two undergoing an additional review cycle. The special issue consists of seven articles that are summarized below. The first two articles also underwent a reproducibity test, whose report is also part of this special issue.

In the first article [20], the authors propose a three-valued extension of Spatio-Temporal Logic and present a statistical model-checking approach to verify properties expressed in this language. Crucially, an undefined truth value is used to account for uncertainty in the simulation. The logic is tested on two case studies, one about threat monitoring and the other one about privacy preservation.

The next article [22] discusses a strategy to abstract stochastic models in the context of chemical reaction networks. The idea of the authors is to replace a complex stochastic model by a simpler one, partially learned from simulations of the complex model. The proposed scenario for the application of this technique is multi-scale modelling. In this context, they discuss two case studies, showing an effective reduction in complexity without losing too much accuracy.

The third article [24] presents a numerical method integrating simulation and numerical solutions for the steady-state of Generalized Petri Nets. The method is based on storing a family of particles and increasing or decreasing them at run-time to adapt to model complexity.

The fourth article [25] is concerned with the efficiency of statistical model checking. More specifically, the authors discuss several probabilistic bounds to be used in sequential schemes to control the number of simulation runs needed, proposing to use Massart bounds to reduce sample size.

The fifth contribution [26] considers the problem of detecting malicious behavior in a building with an access control system. The authors propose to use log data to learn a movement model

(C) 2019 Copyright held by the owner/author(s).

1049-3301/2019/11-ART19

https://doi.org/10.1145/3363784 
inside the building, integrating it with information about the building layout to identify anomalous behavior.

In the sixth article [27], the authors discuss the problem of robust multi-objective synthesis and Pareto front approximation for interval Markov Decision Processes. They discuss the problem complexity, provide an approximation algorithm, and evaluate it against several examples.

The last article [28] is concerned with parametric Continuous Time Markov Chains with alarms, which are events with general parametric distributions. In particular, authors are concerned with the synthesis problem, achieved via a model reduction to a Markov Decision Process and discretization of the parameter space. A crucial step for computational viability is to use a symbolic representation of the so-obtained action space.

\section{REFERENCES}

[1] Christel Baier, Clemens Dubslaff, Luboŝ Korenciiak, Antonín Kuĉera, and Vojtêch Rêhák. 2019. Mean-payoff optimization in continuous-time Markov chains with parametric alarms. ACM Trans. Model. Comput. Simul. 29, 4 (2019).

[2] Carmen Cheh, Uttam Thakore, Ahmed Fawaz, Binbin Chen, William Temple, and William Sanders. 2019. Data-driven model-based detection of malicious insiders via physical access logs. ACM Trans. Model. Comput. Simul. 29, 4 (2019).

[3] Ernst Hahn, Vahid Hashemi, Holger Hermanns, Morteza Lahijanian, and Andrea Turrini. 2019. Interval Markov decision processes with multiple objectives: From robust strategies to Pareto curves. ACM Trans. Model. Comput. Simul. 29, 4 (2019).

[4] Cyrille Jegourel, Jun Sun, and Jin Song Dong. 2019. Sequential schemes for frequentist estimation of properties in statistical model checking. ACM Trans. Model. Comput. Simul. 29, 4 (2019).

[5] Ludovica Luisa Vissat, Michele Loreti, Laura Nenzi, Jane Hillston, and Glenn Marion. 2019. Analysis of spatio-temporal properties of stochastic systems using TSTL. ACM Trans. Model. Comput. Simul. 29, 4 (2019).

[6] Michalis Michaelides, Jane Hillston, and Guido Sanguinetti. 2019. Statistical abstraction for multi-scale spatio-temporal systems. ACM Trans. Model. Comput. Simul. 29, 4 (2019).

[7] Armin Zimmermann and Thomas Hotz. 2019. Integrating simulation and numerical analysis in the evaluation of generalized stochastic Petri nets. ACM Trans. Model. Comput. Simul. 29, 4 (2019).

Luca Bortolussi Nathalie Bertrand

Guest Editors 